\title{
Comparisons of Short-Prefix Based Channel Estimation in Single-Carrier Communication Systems
}

\author{
Huilei Li, Shiwen Fan, Lisha Gong, Guobing Cheng, Shaoqian Li \\ National Key Laboratory of Science and Technology on Communications, \\ University of Electronic Science and Technology of China, Chengdu, China \\ Email: leehuilei@163.com
}

Received August, 2013

\begin{abstract}
In this paper, we compare the performance between channel estimation based on compressed sensing (CS) and time-domain least square (LS) for single-carrier (SC) communication system. Unlike the conventional channel estimation techniques such as frequency domain LS which is used in the condition that the length of pilot sequence is equal to data sequence, the estimation scheme based on CS requires smaller length of pilot sequence. In this paper, the corresponding system structure is presented. Zadoff-Chu sequence is used to generate the pilot sequence, which is shown to perform better in forming measurement matrix of CS than pseudo random sequence. Simulation results demonstrate that channel estimation based on CS achieves a better bit error rate (BER) performance than time domain LS with a smaller pilot sequence and thus raising data rate of the SC communication system.
\end{abstract}

Keywords: Compressed Sensing; Time Domain Least Square; Single-carrier Communication System; Zadoff-Chu Sequence

\section{Introduction}

Recently the single-carrier with frequency domain equalization (SC-FDE) communication system has drawn a great attention as an alternative to the orthogonal frequency division multiplexing (OFDM) communication system, especially in the uplink communications, because it alleviates carrier synchronization problem and the peak-to-average power ratio (PAPR) of transmitted sequences is much lower than that of OFDM[1].

SC-FDE system requires precise channel state information (CSI) for the equalization. To estimate the CSI, the pilot blocks of SC-FDE are inserted between several data blocks in time domain. With the assumption that there are only $L$ number of channel taps where $L$ is smaller than the length of cyclic prefix (CP), the traditional time domain LS estimation can be improved to be used in the condition that the length of pilot sequence is smaller than data sequence but still larger than cyclic prefix.

In this paper, we compare channel estimation based on compressed sensing (CS) and time-domain least square (LS) for the SC communication system. The length of pilot sequence of the compressed method can be smaller than cyclic prefix and can thus raising the data rate of the system. By choosing a good pilot sequence and cyclic prefix to form the CS measurement matrix, we can obtain the sparse channel impulse response (CIR) vector.
The rest of the paper is organized as follows. We start in Section 2 by describing the system model. In Section 3, we review traditional frequency domain LS channel estimation and time domain LS channel estimation which suits the condition that the length of pilot sequence is smaller than data sequence and larger than cyclic prefix. In Section 4, we introduce the idea of compressed channel estimation requiring the length of pilot sequence even smaller than cyclic prefix. Simulation results are given in Section 5 and the paper is concluded in Section 6.

\section{System Model}

Figure 1 shows the block diagram of the transmitter and receiver of the considered SC communication system.

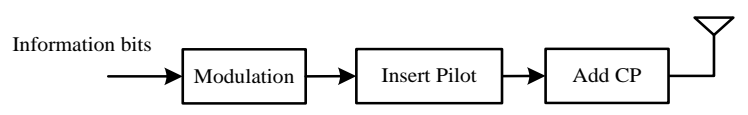

(a) Transmitter

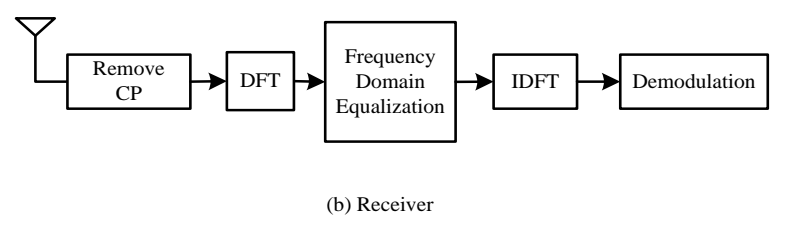

Figure 1. The considered SC communication system. 
We consider the single-carrier block transmission over the multipath Rayleigh fading channel with additive white Gaussian noise. At the transmitter, information bits are mapped into data symbols depending on the modulation type. Each length- $N$ data symbols appended with a length- $N_{c p}$ cyclic prefix forms a data block. The cyclic prefix is assumed to be larger than the known channel impulse response length $L$ to eliminate inter-block interference (IBI). This is achieved by discarding the first $N_{c p}$ received symbols corresponding to the cyclic prefix [2]. The pilot block is formed the same way as the data block while the pilot sequence of each pilot block is of length $N_{p}$. After inserting pilot and adding cyclic prefix, the transmit block is generated. Figure $\mathbf{2}$ shows the transmit block format of the system.

As depicted in Figure 2, the transmit block consists of pilot blocks and data blocks. Pilot sequence

$$
\left\{p(0), p(1), \ldots, p\left(N_{p}-1\right)\right\}
$$

added by a length- $N_{c p}$ CP forms the pilot block and $\left\{x_{i}(0), x_{i}(1), \ldots, x_{i}(N-1)\right\}, i=1, \ldots, K$, each with the same length of $\mathrm{CP}$ as the pilot block, forms $\mathrm{K}$ data blocks. At the receiver, after removing $\mathrm{CP}$, channel estimation, frequency domain equalization which is the frequency domain analog of what is done by a conventional time domain equalizer[3], we obtain the estimated data symbols. By demodulation, these symbols are changed into the estimated information bits.

\section{Channel Estimation of the Pilot Sequence Based on Least-square Channel Estimation}

In the case that the length of pilot sequence is larger than cyclic prefix, i.e. $N_{p}>N_{c p}$, the cyclic prefix of pilot block are copies of the last $N_{c p}$ symbols of the pilot sequence.

At receiver, after removing the cyclic prefix, the inputoutput relationship of the pilot sequence can be expressed in matrix form as follows:

$$
y_{p}=h_{p} x_{p}+n_{p},
$$

$y_{p}, x_{p}$, and $n_{p}$ are length- $N_{p}$ sequence of received, input, and noise symbols, respectively. Note that the $N_{p} \times N_{p}$ channel matrix $h_{p}$ is circulant with first column equal to the channel impulse response (CIR) appended by ( $\left.N_{p}-L\right)$ zeros, therefore it can be diagonalized by the orthonormal discrete Fourier trans-

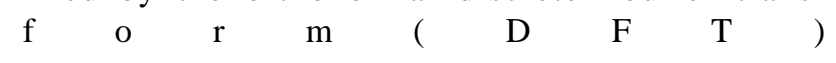

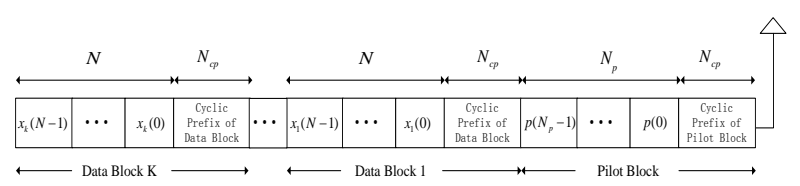

Figure 2. Block format of the considered system. matrices where the diagonal elements are the DFTs of the channel impulse response. That is,

$$
h_{p}=F^{*} H_{p} F=F^{*}\left[\begin{array}{cccc}
H_{0} & 0 & \cdots & 0 \\
0 & H_{1} & \cdots & 0 \\
0 & 0 & \ddots & 0 \\
0 & 0 & \cdots & H_{N_{p}-1}
\end{array}\right] F
$$

where (.)* denotes complex-conjugate transpose; $F$ is the DFT matrix whose element is givenby

$$
F F(i, k)=\frac{1}{\sqrt{N_{p}}} e^{-j 2 \pi(i-1)(k-1) / N_{p}}, 1 \leq i, k \leq N_{p} .
$$

\subsection{Traditional Frequency Domain LS Channel Estimation}

In the case that the length of pilot sequence is equal to that of data sequence, i.e. $N_{p}=N$, after removing сусlic prefix, the received time-domain pilot sequence $y_{p}$ is transformed to the frequency domain by applying DFT to (1)

$$
Y_{p}=F y_{p}=F h_{p} x_{p}+F n_{p}=H_{p} X_{p}+\widetilde{N}_{p},
$$

$Y_{p}, X_{p}$ and $\widetilde{N}_{p}$ are length- $N_{p}$ frequency-domain response of received, input, and noise symbols, respectively.

Therefore, the traditional LS channel estimation of $H_{p}$ at the pilot sequenceis

$$
\widehat{H}_{p_{-} L S}=\frac{Y_{p}}{X_{p}},
$$

\subsection{Time Domain Least-square Channel Estimation}

Using the time domain LS channel estimation, the length of pilot sequence can be reduced to be smaller than data sequence, i.e. $N_{p}<N$. Therefore, the equation (1)-(3) are still satisfied.

With the assumption that the channel impulse response length is smaller than $N_{c p}$ [4], the input-output relationship of the system in frequency domain can be expressed as

$$
Y_{p}=\operatorname{diag}\left(X_{p}\right) F_{p} h+\widetilde{N}_{p}
$$

where $\operatorname{diag}\left(X_{p}\right)$ stands for diagonal matrix with the column vector $X_{p}$ on its diagonal; $F_{p}$ is an $N_{p} \times N_{c p}$ matrix which retains only the first $N_{c p}$ columns of $F$; and $h$ is a length- $N_{c p}$ column vector which retains the first $N_{c p}$ elements of $h_{p}$. Then the improved LS channel estimation is

$$
\hat{h}_{p_{-} L S}=\left(Q^{H} Q\right)^{-1} Q^{H} Y_{p}
$$


where

$$
Q=\operatorname{diag}\left(X_{p}\right) F_{p},
$$

Then, by doing $N$-points DFT of $\hat{h}_{p_{-} L S}$, we obtain the estimated channel frequency response $\widehat{H}_{p_{-} L S}$ of the pilot sequence. That is,

$$
\widehat{H}_{p_{-} L S}=F_{N} \hat{h}_{p_{-} L S},
$$

where $F_{N}(i, k)=e^{-j 2 \pi(i-1)(k-1) / N}, 1 \leq i \leq N, 1 \leq k \leq N_{c p}$.

\section{Channel Estimation of the Pilot Sequence Based on Compressed Sensing}

When the length ofpilot sequence is not smaller than that of cyclic prefix, i.e. $N_{p} \geq N_{c p}$, the cyclic prefix of the pilot sequence is the copy of the last $N_{c p}$ symbols of the pilot sequence, and then (1) is equal to

$$
y_{p}=T_{p} h+n_{p},
$$

where

$$
T_{p}=\left[\begin{array}{cccc}
x_{0} & x_{N_{p}-1} & \cdots & x_{N_{p}-N_{c p}+1} \\
x_{1} & x_{0} & \cdots & x_{N_{p}-N_{c p}+2} \\
\vdots & \vdots & \vdots & \vdots \\
\vdots & \vdots & \vdots & \vdots \\
x_{N_{p}-1} & x_{N_{p}-2} & \cdots & x_{N_{p}-N_{c p}}
\end{array}\right],
$$

is a $N_{p} \times N_{c p}$ Toeplitz matrix formed by the pilot sequence.

Now we consider the case that the length of the pilot sequence is smaller than cyclic prefix, i.e. $N_{p}<N_{c p}$. Assuming the cyclic prefix of the pilot block in the form of vector is $\left[c_{0}, c_{1}, \ldots, c_{N_{c p}-1}\right]^{T}$, then the Toeplitz matrix $T_{p}$ in (9) can be modified to be

$$
T_{p}=\left[\begin{array}{cccccc}
x_{0} & c_{N_{c p}-1} & \cdots & c_{N_{c p}-N_{p}+1} & \cdots & c_{1} \\
x_{1} & x_{0} & \ddots & \ddots & \ddots & \vdots \\
\vdots & \vdots & \ddots & c_{N_{c p}-1} & \ddots & \vdots \\
x_{N_{p}-1} & x_{N_{p}-2} & \cdots & x_{0} & c_{N_{c p}-1} & c_{N_{C p}-N_{p}+1}
\end{array}\right]
$$

which is a $N_{p} \times N_{c p}$ Toeplitz matrix formed by both the pilot sequence and its cyclic prefix.

It is known that CS can be effectively utilized in linear, time-invariant system identification problems provided the impulse response of the system is (approximately or exactly) sparse. An immediate application is in wireless multipath channel estimation[5]. As the length- $N_{c p}$ CIR $h$ of the sparse wireless channel has only few of its taps nonzero, then using (9) we can change the matter of channel estimation into the matter of recovery of $h$ based on compressed sensing.

$$
y_{p} \approx T_{p} h,
$$

Therefore, $T_{p}$ is considered as the CS measurement matrix. The channel estimation based on CS introduced in this paper uses the orthogonal matching pursuit (OMP) algorithm to solve the problem of compressed sensing.

\subsection{Selection of Pilot Sequence}

Advances in CS show that the sparse $h$ can be reconstructed from the measurement $y_{p}$ with high probability when measurement matrix $T_{p}$ satisfies RIP[6]. However, there is no known method to test in polynomial time whether a given matrix satisfies RIP. An alternative approach we adopt here is to minimize the coherence of $T_{p}$ [7]. The coherence of a $N_{p} \times N_{c p}$ measurement matrix $T_{p}$ is the maximum absolute cross-correlation between the normalized columns of $T_{p}$ :

$$
\mu_{T_{p}}=\max _{\substack{1 \leq 1, k \leq N_{c p} \\ l \neq k}} \frac{\left|\left\langle\varphi_{l}, \varphi_{k}\right\rangle\right|}{\left\|\varphi_{l}\right\|_{2} \cdot\left\|\varphi_{k}\right\|_{2}},
$$

The smaller the coherence $\mu_{T_{p}}$ is, the better the measurement matrix preserves the information of the sparse vector $h$ in the produced samples $y_{p}$ [7].

$T_{p}$ is formed by elements of the pilot block, so the selection of pilot sequence and its cyclic prefix is important. In this paper, we choose Zadoff-Chu sequence which has constant envelope and uniform spectrum[8] to form the pilot sequence and its cyclic prefix. Compared with pseudo random sequence, measurement matrix formed by Chu sequence has smaller $\mu_{T_{p}}$.

A length- $N_{p}$ Chu sequence $\left\{P_{k}\right\}_{k=0}^{N_{p}-1}$ used as the pilot sequence is represented as

$$
P_{k}=\left\{\begin{array}{c}
e^{j \pi r k^{2} / N_{p}, \text { for even } N_{p}} \\
e^{j \pi r k(k+1) / N_{p}, \text { for odd } N_{p}}
\end{array},\right.
$$

where $r$ is relatively prime to $N_{p}$. The length- $N_{c p}$ cyclic prefix of the pilot sequence is generated the same way as the pilot sequence using a length- $N_{c p}$ Chu sequence $\left\{c_{k}\right\}_{k=0}^{N_{c p}-1}$.

Simulation results show that measurement matrix formed by Chu sequence performs better in the compressed channel estimation than pseudo random sequence.

\subsection{Proposed Compressed Channel Estimation}

In this paper, the compressed channel estimation is based on OMP algorithm. OMP is an iterative greedy algorithm that selects at each step the column, which is most correlated with the current residuals[9]. With OMP, faster 
convergence to a sparse solution is obtained by eliminating the re-selection problem[10].

For (9), with the knowledge of the received time domain pilots $y_{p}$ and the Toeplitz matrix $T_{p}$ formed by pilot block, we use OMP algorithm to estimate the positions and values of the taps of the sparse vector $h$ and then transform it into frequency domain the same way as (8).

\section{Simulation Results}

In this paper, we do simulations to compare the performance of traditional LS channel estimation, time domain LS channel estimation and the compressed channel estimation. Suppose the coherent time of the sparse single-carrier channel lasts about 10 data blocks, the number of nonzero taps of the channel is $L=6$, the length of each data sequence is $\mathrm{N}=256$ and the length of CP for both the pilot sequence and data sequence is $N_{c p}=64$. The transmission block contains 12 data blocks and 3 pilot blocks inserted between each 6 data blocks. Information about the Rayleigh channel of the system is described in Table 1 with additive white Guassian noise. We assume that the time delays of the channel are integral times of the system sampling period $T_{s}=9.7657 e-07 \mathrm{~s}$ for convenience.

At the receiver, we apply first order linear interpolation to gain the channel of data sequence in frequency domain and Minimum mean square error (MMSE) equalization as the equalization algorithm. Let $N_{p}=m$ and consider the following groups of simulations:

1) We apply traditonal LS channel estimation on the pilot block for $m=256$;

2) We apply time domain LS channel estimation on the pilot block for $m=128,64$;

3) We apply the compressed channel estimation on the pilot block for $m=128,64,32$;

The BER performance results of the methodsabove are given by Figure 3. "FLS" means traditional LS channel estimation, "TLS” means time domain LS channel estimation, and "CS" means the estimation method based on compressed sensing. "H_ideal” means ideal channel estimation.

Table 1. Parameters of the multipath Rayleigh channel.

\begin{tabular}{ccc}
\hline \multirow{2}{*}{ Path } & \multicolumn{2}{c}{ Table Column Head } \\
\cline { 2 - 3 } & Delay Time & Average Power $(\mathbf{d B})$ \\
\hline 1 & 0 & 0 \\
2 & $2 T_{s}$ & -3 \\
3 & $8 T_{s}$ & -6 \\
4 & $16 T_{s}$ & -9 \\
\hline
\end{tabular}

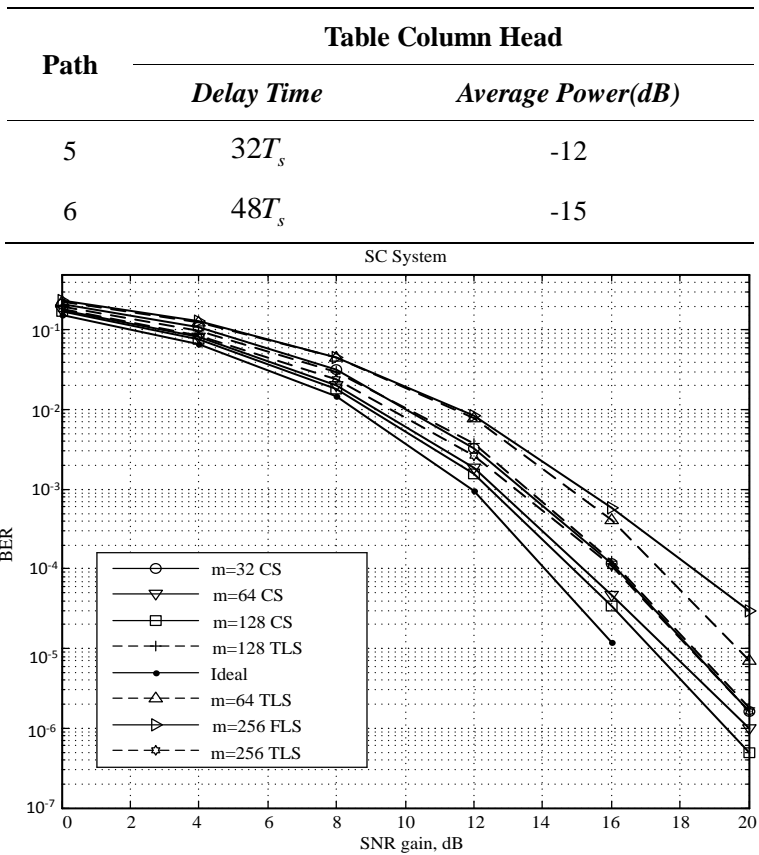

Figure 3. BER performances of the methods above.

Figure 3 shows that by using time domain LS method or the compressed method, the BER performance of the system can be improved compared with traditional LS method even reducing the length of the pilot sequence. For time domain LS method, the larger the length of pilot sequence is, the better its BER performance is. For the compressed method, when the lengthof pilot sequence is larger than or equal to its cyclic prefix, it performs better than time domain LS method. As the length of pilot sequence reduces to $m=32$ which is smaller than its cyclic prefix, the BER performance of thecompressed channel estimation is almost the same with time domain LS channel estimation with $m=256$.

\section{Conclusions}

In this paper, a comparison among channel estimation based on traditional LS method, time domain LS and compressed sensing in SC communication system has been done for the sparse channel. We showed that among these three estimation methods, both time domain LS and compressed method perform better than traditional LS method. Moreover, the compressed method based on OMP algorithm reduces the length of pilot sequence required for channel estimation compared with time domain LS method without performance degradation. Simulation results showed that the compressed method has significant performance gains over time domain LS estimation method under the same condition of the length of pilot sequence.

\section{Acknowledgements}


This work is supported in part by the National Science Foundation of China under Grant number 61101101, National Grand Special Science and Technology Project of China under Grant No. 2010ZX03006-002-02, Program for New Century Excellent Talents in University of China ((NCET110058), the Foundation Project of National Key Laboratory of Science and Technology on Communications under Grant 9140C020404120C0201, and Key Laboratory of Universal Wireless Communications, Beijing university of Posts and Telecommunications, Ministry of Education, P.R.China (No. KFKT2012102).

\section{REFERENCES}

[1] D. Kim, U.-K. Kwon and G.-H. Im, "Pilot Positon Selection and Detection for Channel Estimation of SC-FDE," IEEE Communications Letter, Vol. 12, No. 5, 2008, pp. 350-352. doi:10.1109/LCOMM.2008.080071

[2] N. Al-Dhahir, "Single-Carrier Frequency-Domain Equalization for Space-Time Block-Coded Transmissions Over Frequency-Selective Fading Channels," IEEE Communication Letters, Vol. 5, No. 7, 2001, pp. 304-306. doi: $10.1109 / 4234.935750$

[3] D. Falconer, S. L. Ariyavisitakul, A. Benyamin-Seeyar and B. Eidson, "Frequency Domain Equalization for Single-Carrier Broadband Wireless Systems,” IEEE Communication Magazine, 2002, pp. 58-66. doi:10.1109/35.995852

[4] M. K. Ozdemir and H. Arslan, "Channel Estimation for
Wireless OFDM Systems," IEEE Communications Surveys \& Tutorials, Vol. 9, No. 2, pp. 18-48, 2nd Quarter, 2007.

[5] J. Haupt, W. U. Bajwa, G. Raz and R. Nowak, “Toeplitz Compressed Sensing Matrices with Applications to Sparse Channel Estimation," IEEE Transaction on Information Theory, Vol. 56, No. 11, 2010, pp. 5862-5875. doi:10.1109/TIT.2010.2070191

[6] E. J. Candes and T. Tao, "Near-optimal Signal Recovery from Random Projections: Univeral Encoding Strategies,” IEEE Transaction on Information Theory, Vol. 52, No. 12, 2006, pp. 5406-5425. doi:10.1109/TIT.2006.885507

[7] C. H. Qi and L. N. Wu, "A Study of Deterministic Pilot Allocation for Sparse Channel Estimation in OFDM System,” IEEE Communication Letters, Vol. 16, No. 5, 2012, pp. 742-744, May, 2012. doi:10.1109/LCOMM.2012.032612.112553

[8] C.-T. Lam and D. D. Falconer, F. Danilo-Lemoine and R. Dinis, "Channel Estimation for SC-FDE Systems Using Frequency Domain Multiplexed Pilots,” Vehicular Technology Conference, 2006, pp. 1-5.

[9] T. Tony Cai and Lie Wang, "Orthogonal Matching Pursuit for Sparse Signal Recovery With Noise,” IEEE Transactions on Information Theory, Vol. 57, No. 7, 2011, pp. 4680-4688.doi:10.1109/TIT.2011.2146090

[10] G. Z. Karabulut, A. Yongacoglu, "Sparse Channel Estimation using Orthogonal Matching Pursuit Algorithm," Vehicular Technology Conference, pp. 3880-3884, IEEE, 2004. 